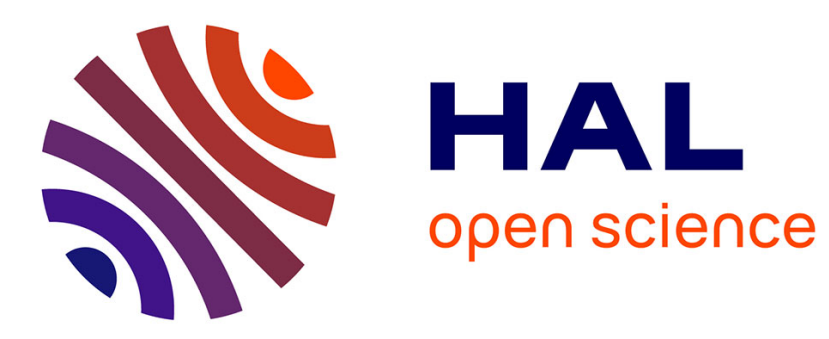

\title{
Multiseed Lossless Filtration
}

Gregory Kucherov, Laurent Noé, Mikhail A. Roytberg

\section{To cite this version:}

Gregory Kucherov, Laurent Noé, Mikhail A. Roytberg. Multiseed Lossless Filtration. IEEE/ACM Transactions on Computational Biology and Bioinformatics, 2005, 2 (1), pp.51-61. 10.1109/TCBB.2005.12 . inria-00354810

\section{HAL Id: inria-00354810 https://hal.inria.fr/inria-00354810}

Submitted on 21 Jan 2009

HAL is a multi-disciplinary open access archive for the deposit and dissemination of scientific research documents, whether they are published or not. The documents may come from teaching and research institutions in France or abroad, or from public or private research centers.
L'archive ouverte pluridisciplinaire HAL, est destinée au dépôt et à la diffusion de documents scientifiques de niveau recherche, publiés ou non, émanant des établissements d'enseignement et de recherche français ou étrangers, des laboratoires publics ou privés. 


\title{
Multiseed lossless filtration
}

\author{
Gregory Kucherov, Laurent Noé, Mikhail Roytberg
}

\begin{abstract}
We study a method of seed-based lossless filtration for approximate string matching and related bioinformatics applications. The method is based on a simultaneous use of several spaced seeds rather than a single seed as studied by Burkhardt and Karkkainen [1]. We present algorithms to compute several important parameters of seed families, study their combinatorial properties, and describe several techniques to construct efficient families. We also report a large-scale application of the proposed technique to the problem of oligonucleotide selection for an EST sequence database.
\end{abstract}

\section{Index Terms}

filtration, string matching, gapped seed, gapped Q-gram, local alignment, sequence similarity, seed family, multiple spaced seeds, dynamic programming, EST, oligonucleotide selection.

\section{INTRODUCTION}

$\mathbf{F}$

ILTERING is a widely-used technique in biosequence analysis. Applied to the approximate string matching problem [2], it can be summarized by the following two-stage scheme: to find approximate occurrences (matches) of a given string in a sequence (text), one first quickly discards (filters out) those sequence regions where matches cannot occur, and then checks out the remaining parts of the sequence for actual matches. The filtering is done according to small patterns of a specified form that the searched string is assumed to share, in the exact way, with its approximate occurrences. A similar filtration scheme is used by heuristic local alignment algorithms ([3], [曰], [5], [6], to mention a few): they first

An extended abstract of this work has been presented to the Combinatorial Pattern Matching conference (Istanbul, July 2004)

Gregory Kucherov and Laurent Noé are with the INRIA/LORIA, 615, rue du Jardin Botanique, B.P. 101, 54602 Villerslès-Nancy, France, e-mail: [Gregory.Kucherov, Laurent.Noe] @loria.fr

Mikhail Roytberg is with the Institute of Mathematical Problems in Biology, Pushchino, Moscow Region, Russia, e-mail: roytberg@impb.psn.ru 
identify potential similarity regions that share some patterns and then actually check whether those regions represent a significant similarity by computing a corresponding alignment.

Two types of filtering should be distinguished - lossless and lossy. A lossless filtration guarantees to detect all sequence fragments under interest, while a lossy filtration may miss some of them, but still tries to detect a majority of them. Local alignment algorithms usually use a lossy filtration. On the other hand, the lossless filtration has been studied in the context of approximate string matching problem [7], [1]. In this paper, we focus on the lossless filtration.

In the case of lossy filtration, its efficiency is measured by two parameters, usually called selectivity and sensitivity. The sensitivity measures the part of sequence fragments of interest that are missed by the filter (false negatives), and the selectivity indicates what part of detected candidate fragments don't actually represent a solution (false positives). In the case of lossless filtration, only the selectivity parameter makes sense and is therefore the main characteristic of the filtration efficiency.

The choice of patterns that must be contained in the searched sequence fragments is a key ingredient of the filtration algorithm. Gapped seeds (spaced seeds, gapped $q$-grams) have been recently shown to significantly improve the filtration efficiency over the "traditional" technique of contiguous seeds. In the framework of lossy filtration for sequence alignment, the use of designed gapped seeds has been introduced by the PATTERNHUNTER method [ 4 ] and then used by some other algorithms (e.g. [5], [6]). In [8], [9], spaced seeds have been shown to improve indexing schemes for similarity search in sequence databases. The estimation of the sensitivity of spaced seeds (as well as of some extended seed models) has been subject of several recent studies [10], [11], [12], [13], [14], [15]. In the framework of lossless filtration for approximate pattern matching, gapped seeds were studied in [1] (see also [7]) and have also been shown to increase the filtration efficiency considerably.

In this paper, we study an extension of the lossless single-seed filtration technique [1]. The extension is based on using seed families rather than individual seeds. The idea of simultaneous use of multiple seeds for DNA local alignment was already envisaged in [4] and applied in PATTERnHUnter II software [16]. The problem of designing efficient seed families has also been studied in [17]. In [18], multiple seeds have been applied to the 
protein search. However, the issues analysed in the present paper are quite different, due to the proposed requirement for the search to be lossless.

The rest of the paper is organized as follows. After formally introducing the concept of multiple seed filtering in Section [I. Section IIII is devoted to dynamic programming algorithms to compute several important parameters of seed families. In Section IV, we first study several combinatorial properties of families of seeds, and, in particular, seeds having a periodic structure. These results are used to obtain a method for constructing efficient seed families. We also outline a heuristic genetic programming algorithm for constructing seed families. Finally, in Section $\square$, we present several seed families we computed, and we report a large-scale experimental application of the method to a practical problem of oligonucleotide selection.

\section{Multiple SEED FILTERING}

A seed $Q$ (called also spaced seed or gapped q-gram) is a list $\left\{p_{1}, p_{2}, \ldots, p_{d}\right\}$ of positive integers, called matching positions, such that $p_{1}<p_{2}<\ldots<p_{d}$. By convention, we always assume $p_{1}=0$. The span of a seed $Q$, denoted $s(Q)$, is the quantity $p_{d}+1$. The number $d$ of matching positions is called the weight of the seed and denoted $w(Q)$. Often we will use a more visual representation of seeds, adopted in [1], as words of length $s(Q)$ over the two-letter alphabet $\{\#,-\}$, where \# occurs at all matching positions and - at all positions in between. For example, seed $\{0,1,2,4,6,9,10,11\}$ of weight 8 and span 12 is represented by word \#\#\#-\#-\#--\#\#\#. The character - is called a joker. Note that, unless otherwise stated, the seed has the character \# at its first and last positions.

Intuitively, a seed specifies the set of patterns that, if shared by two sequences, indicate a possible similarity between them. Two sequences are similar if the Hamming distance between them is smaller than a certain threshold. For example, sequences CACTCGT and CACACTT are similar within Hamming distance 2 and this similarity is detected by the seed $\# \#-\#$ at position 2. We are interested in seeds that detect all similarities of a given length with a given Hamming distance.

Formally, a gapless similarity (hereafter simply similarity) of two sequences of length $m$ is a binary word $w \in\{0,1\}^{m}$ interpreted as a sequence of matches (1's) and mismatches (0's) of individual characters from the alphabet of input sequences. A seed $Q=\left\{p_{1}, p_{2}, \ldots, p_{d}\right\}$ 
matches a similarity $w$ at position $i, 1 \leq i \leq m-p_{d}+1$, iff for every $j \in[1 . . d]$, we have $w\left[i+p_{j}\right]=1$. In this case, we also say that seed $Q$ has an occurrence in similarity $w$ at position $i$. A seed $Q$ is said to detect a similarity $w$ if $Q$ has at least one occurrence in $w$.

Given a similarity length $m$ and a number of mismatches $k$, consider all similarities of length $m$ containing $k 0$ 's and $(m-k)$ 1's. These similarities are called $(m, k)$-similarities. A seed $Q$ solves the detection problem $(m, k)$ (for short, the $(m, k)$-problem) iff all of $\left(\begin{array}{c}m \\ k\end{array}\right)$ $(m, k)$-similarities $w$ are detected by $Q$. For example, one can check that seed \#-\#\#--\#-\#\# solves the $(15,2)$-problem.

Note that the weight of the seed is directly related to the selectivity of the corresponding filtration procedure. A larger weight improves the selectivity, as less similarities will pass through the filter. On the other hand, a smaller weight reduces the filtration efficiency. Therefore, the goal is to solve an $(m, k)$-problem by a seed with the largest possible weight.

Solving $(m, k)$-problems by a single seed has been studied by Burkhardt and Kärkkäinen [1]. An extension we propose here is to use a family of seeds, instead of a single seed, to solve the $(m, k)$-problem. Formally, a finite family of seeds $F=<Q_{l}>_{l=1}^{L}$ solves an $(m, k)$-problem iff for any $(m, k)$-similarity $w$, there exists a seed $Q_{l} \in F$ that detects $w$.

Note that the seeds of the family are used in the complementary (or disjunctive) fashion, i.e. a similarity is detected if it is detected by one of the seeds. This differs from the conjunctive approach of [7] where a similarity should be detected by two seeds simultaneously.

The following example motivates the use of multiple seeds. In [1], it has been shown that a seed solving the $(25,2)$-problem has the maximal weight 12 . The only such seed

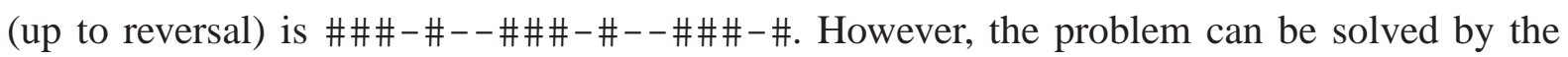

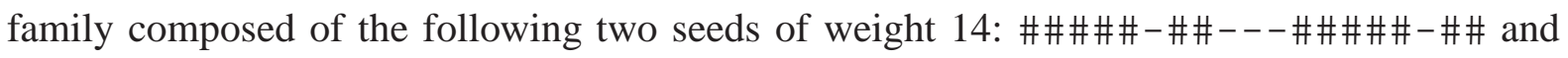
\#-\#\#---\#\#\#\#\#-\#\#---\#\#\#\#.

Clearly, using these two seeds increases the selectivity of the search, as only similarities having 14 or more matching characters pass the filter vs 12 matching characters in the case of single seed. On uniform Bernoulli sequences, this results in the decrease of the number of candidate similarities by the factor of $|A|^{2} / 2$, where $A$ is the input alphabet. This illustrates the advantage of the multiple seed approach: it allows to increase the selectivity while preserving a lossless search. The price to pay for this gain in selectivity is multiplying 
the work on identifying the seed occurrences. In the case of large sequences, however, this is largely compensated by the decrease in the number of false positives caused by the increase of the seed weight.

\section{COMPUTING PROPERTIES OF SEED FAMILIES}

Burkhardt and Kärkkäinen [1] proposed a dynamic programming algorithm to compute the optimal threshold of a given seed - the minimal number of its occurrences over all possible $(m, k)$-similarities. In this section, we describe an extension of this algorithm for seed families and, on the other hand, describe dynamic programming algorithms for computing two other important parameters of seed families that we will use in a later section.

Consider an $(m, k)$-problem and a family of seeds $F=<Q_{l}>_{l=1}^{L}$. We need the following notation.

- $s_{\max }=\max \left\{s\left(Q_{l}\right)\right\}_{l=1}^{L}, s_{\min }=\min \left\{s\left(Q_{l}\right)\right\}_{l=1}^{L}$,

- for a binary word $w$ and a seed $Q_{l}, \operatorname{suff}\left(Q_{l}, w\right)=1$ if $Q_{l}$ matches $w$ at position $(|w|-$ $\left.s\left(Q_{l}\right)+1\right)$ (i.e. matches a suffix of $w$ ), otherwise $\operatorname{suff}\left(Q_{l}, w\right)=0$,

- $\operatorname{last}(w)=1$ if the last character of $w$ is 1 , otherwise $\operatorname{last}(w)=0$,

- $z \operatorname{eros}(w)$ is the number of 0's in $w$.

\section{A. Optimal threshold}

Given an $(m, k)$-problem, a family of seeds $F=<Q_{l}>_{l=1}^{L}$ has the optimal threshold $T_{F}(m, k)$ if every $(m, k)$-similarity has at least $T_{F}(m, k)$ occurrences of seeds of $F$ and this is the maximal number with this property. Note that overlapping occurrences of a seed as well as occurrences of different seeds at the same position are counted separately. For example, the singleton family $\{\# \# \#-\# \#\}$ has threshold 2 for the $(15,2)$-problem.

Clearly, $F$ solves an $(m, k)$-problem if and only if $T_{F}(m, k)>0$. If $T_{F}(m, k)>1$, then one can strengthen the detection criterion by requiring several seed occurrences for a similarity to be detected. This shows the importance of the optimal threshold parameter.

We now describe a dynamic programming algorithm for computing the optimal threshold $T_{F}(m, k)$. For a binary word $w$, consider the quantity $T_{F}(m, k, w)$ defined as the minimal number of occurrences of seeds of $F$ in all $(m, k)$-similarities which have the suffix $w$. By definition, $T_{F}(m, k)=T_{F}(m, k, \varepsilon)$. Assume that we precomputed values $\mathcal{T}_{F}(j, w)=$ 
$T_{F}\left(s_{\max }, j, w\right)$, for all $j \leq \max \left\{k, s_{\max }\right\},|w|=s_{\max }$. The algorithm is based on the following recurrence relations on $T_{F}(i, j, w)$, for $i \geq s_{\max }$.

$$
T_{F}(i, j, w[1 . . n])= \begin{cases}\mathcal{T}_{F}(j, w), & \text { if } i=s_{\text {max }}, \\ T_{F}(i-1, j-1, w[1 . . n-1]), & \text { if } w[n]=0, \\ T_{F}(i-1, j, w[1 . . n-1])+\left[\sum_{l=1}^{L} \operatorname{suff}\left(Q_{l}, w\right)\right], & \text { if } n=s_{\text {max }}, \\ \min \left\{T_{F}(i, j, 1 . w), T_{F}(i, j, 0 . w)\right\}, & \text { if zeros }(w)<j, \\ T_{F}(i, j, 1 . w), & \text { if zeros }(w)=j .\end{cases}
$$

The first relation is an initial condition of the recurrence. The second one is based on the fact that if the last symbol of $w$ is 0 , then no seed can match a suffix of $w$ (as the last position of a seed is always assumed to be a matching position). The third relation reduces the size of the problem by counting the number of suffix seed occurrences. The fourth one splits the counting into two cases, by considering two possible characters occurring on the left of $w$. If $w$ already contains $j 0$ 's, then only 1 can occur on the left of $w$, as stated by the last relation.

A dynamic programming implementation of the above recurrence allows to compute $T_{F}(m, k, \varepsilon)$ in a bottom-up fashion, starting from initial values $\mathcal{T}_{F}(j, w)$ and applying the above relations in the order in which they are given. A straightforward dynamic programming implementation requires $O\left(m \cdot k \cdot 2^{\left(s_{\max }+1\right)}\right)$ time and space. However, the space complexity can be immediately improved: if values of $i$ are processed successively, then only $O\left(k \cdot 2^{\left(s_{\max }+1\right)}\right)$ space is needed. Furthermore, for each $i$ and $j$, it is not necessary to consider all $2^{\left(s_{\max }+1\right)}$ different strings $w$, but only those which contain up to $j 0$ 's. The number of those $w$ is $g\left(j, s_{\max }\right)=\sum_{e=0}^{j}\left(\begin{array}{c}s_{\max } \\ e\end{array}\right)$. For each $i, j$ ranges from 0 to $k$. Therefore, for each $i$, we need to store $f\left(k, s_{\max }\right)=\sum_{j=0}^{k} g\left(j, s_{\max }\right)=\sum_{j=0}^{k}\left(\begin{array}{c}s_{\max } \\ j\end{array}\right) \cdot(k-j+1)$ values. This yields the same space complexity as for computing the optimal threshold for one seed [1]].

The quantity $\sum_{l=1}^{L} \operatorname{suff}\left(Q_{l}, w\right)$ can be precomputed for all considered words $w$ in time $O\left(L \cdot g\left(k, s_{\max }\right)\right)$ and space $O\left(g\left(k, s_{\max }\right)\right)$, under the assumption that checking an individual match is done in constant time. This leads to the overall time complexity $O\left(m \cdot f\left(k, s_{\max }\right)+\right.$ $\left.L \cdot g\left(k, s_{\max }\right)\right)$ with the leading term $m \cdot f\left(k, s_{\max }\right.$ ) (as $L$ is usually small compared to $m$ and $g\left(k, s_{\max }\right)$ is smaller than $\left.f\left(k, s_{\max }\right)\right)$. 


\section{B. Number of undetected similarities}

We now describe a dynamic programming algorithm that computes another characteristic of a seed family, that will be used later in Section IV-D. Consider an $(m, k)$-problem. Given a seed family $F=<Q_{l}>_{l=1}^{L}$, we are interested in the number $U_{F}(m, k)$ of $(m, k)$-similarities that are not detected by $F$. For a binary word $w$, define $U_{F}(m, k, w)$ to be the number of undetected $(m, k)$-similarities that have the suffix $w$.

Similar to [10], let $X(F)$ be the set of binary words $w$ such that $(i)|w| \leq s_{\max }$, (ii) for any $Q_{l} \in F$, suff $\left(Q_{l}, 1^{s_{\max }-|w|} w\right)=0$, and (iii) no proper suffix of $w$ satisfies (ii). Note that word 0 belongs to $X(F)$, as the last position of every seed is a matching position.

The following recurrence relations allow to compute $U_{F}(i, j, w)$ for $i \leq m, j \leq k$, and $|w| \leq s_{\max }$

$$
U_{F}(i, j, w[1 . . n])= \begin{cases}\left(\begin{array}{c}
i-|w| \\
j-\operatorname{zeros}(w)
\end{array}\right), & \text { if } i<s_{\text {min }}, \\
0, & \text { if } \exists l \in[1 . . L], \operatorname{suff}\left(Q_{l}, w\right)=1, \\
U_{F}(i-1, j-\operatorname{last}(w), w[1 . . n-1]), & \text { if } w \in X(F), \\
U_{F}(i, j, 1 . w)+U_{F}(i, j, 0 . w), & \text { if } z \operatorname{eros}(w)<j, \\
U_{F}(i, j, 1 . w), & \text { if } \operatorname{zeros}(w)=j .\end{cases}
$$

The first condition says that if $i<s_{\min }$, then no word of length $i$ will be detected, hence the binomial coefficient. The second condition is straightforward. The third relation follows from the definition of $X(F)$ and allows to reduce the size of the problem. The last two conditions are similar to those from the previous section.

The set $X(F)$ can be precomputed in time $O\left(L \cdot g\left(k, s_{\max }\right)\right)$ and the worst-case time complexity of the whole algorithm remains $O\left(m \cdot f\left(k, s_{\max }\right)+L \cdot g\left(k, s_{\max }\right)\right)$.

\section{Contribution of a seed}

Using a similar dynamic programming technique, one can compute, for a given seed of the family, the number of $(m, k)$-similarities that are detected only by this seed and not by the others. Together with the number of undetected similarities, this parameter will be used later in Section IV-D.

Given an $(m, k)$-problem and a family $F=<Q_{l}>_{l=1}^{L}$, we define $S_{F}(m, k, l)$ to be the number of $(m, k)$-similarities detected by the seed $Q_{l}$ exclusively (through one or several 
occurrences), and $S_{F}(m, k, l, w)$ to be the number of those similarities ending with the suffix $w$. A dynamic programming algorithm similar to the one described in the previous sections can be applied to compute $S_{F}(m, k, l)$. The recurrence is given below.

$$
S_{F}(i, j, l, w[1 . . n])= \begin{cases}0 & \text { if } i<s_{\text {min }} \text { or } \exists l^{\prime} \neq l \operatorname{suff}\left(Q_{l^{\prime}}, w\right)=1 \\ S_{F}(i-1, j-1, l, w[1 . . n-1]) & \text { if } w[n]=0 \\ S_{F}(i-1, j, l, w[1 . . n-1]) & \text { if } n=\left|Q_{l}\right| \text { and } \operatorname{suff}\left(Q_{l}, w\right)=0 \\ S_{F}(i-1, j, l, w[1 . . n-1]) & \text { if } n=s_{\text {max }} \text { and } \operatorname{suff}\left(Q_{l}, w\right)=1 \\ +U_{F}(i-1, j, w[1 . . n-1]) & \text { and } \forall l^{\prime} \neq l, \operatorname{suff}\left(Q_{l^{\prime}}, w\right)=0, \\ S_{F}(i, j, l, 1 . w[1 . . n]) & \\ +S_{F}(i, j, l, 0 . w[1 . . n]) & \text { if } z \operatorname{eros}(w)<j \\ S_{F}(i, j, l, 1 . w[1 . . n]) & \text { if } z \operatorname{zeros}(w)=j\end{cases}
$$

The third and fourth relations play the principal role: if $Q_{l}$ does not match a suffix of $w[1 . . n]$, then we simply drop out the last letter. If $Q_{l}$ matches a suffix of $w[1 . . n]$, but no other seed does, then we count prefixes matched by $Q_{l}$ exclusively (term $S_{F}(i-1, j, l, w[1 . . n-1])$ ) together with prefixes matched by no seed at all (term $U_{F}(i-1, j, w[1 . . n-1])$ ). The latter is computed by the algorithm of the previous section.

The complexity of computing $S_{F}(m, k, l)$ for a given $l$ is the same as the complexity of dynamic programming algorithms from the previous sections.

\section{SEED DESIGN}

In the previous Section we showed how to compute various useful characteristics of a given family of seeds. A much more difficult task is to find an efficient seed family that solves a given $(m, k)$-problem. Note that there exists a trivial solution where the family consists of all $\left(\begin{array}{l}m \\ k\end{array}\right)$ position combinations, but this is in general unacceptable in practice because of a huge number of seeds. Our goal is to find families of reasonable size (typically, with the number of seeds smaller than ten), with a good filtration efficiency.

In this section, we present several results that contribute to this goal. In Section IV-A, we start with the case of single seed with a fixed number of jokers and show, in particular, that for one joker, there exists one best seed in a sense that will be defined. We then show in Section IV-B that a solution for a larger problem can be obtained from a smaller one 
by a regular expansion operation. In Section $[\mathrm{IV}-\mathrm{C}$, we focus on seeds that have a periodic structure and show how those seeds can be constructed by iterating some smaller seeds. We then show a way to build efficient families of periodic seeds. Finally, in Section IV-D, we briefly describe a heuristic approach to constructing efficient seed families that we used in the experimental part of this work presented in Section $\square$.

\section{A. Single seeds with a fixed number of jokers}

Assume that we fixed a class of seeds under interest (e.g. seeds of a given minimal weight). One possible way to define the seed design problem is to fix a similarity length $m$ and find a seed that solves the $(m, k)$-problem with the largest possible value of $k$. A complementary definition is to fix $k$ and minimize $m$ provided that the $(m, k)$-problem is still solved. In this section, we adopt the second definition and present an optimal solution for one particular case.

For a seed $Q$ and a number of mismatches $k$, define the $k$-critical length for $Q$ as the minimal value $m$ such that $Q$ solves the $(m, k)$-problem. For a class of seeds $\mathcal{C}$ and a value $k$, a seed is $k$-optimal in $\mathcal{C}$ if $Q$ has the minimal $k$-critical length among all seeds of $\mathcal{C}$.

One interesting class of seeds $\mathcal{C}$ is obtained by putting an upper bound on the possible number of jokers in the seed, i.e. on the number $(s(Q)-w(Q))$. We have found a general solution of the seed design problem for the class $\mathcal{C}_{1}(n)$ consisting of seeds of weight $d$ with only one joker, i.e. seeds $\#^{d-r}-\#^{r}$.

Consider first the case of one mismatch, i.e. $k=1$. A 1 -optimal seed from $\mathcal{C}_{1}(d)$ is $\#^{d-r}-\#^{r}$ with $r=\lfloor d / 2\rfloor$. To see this, consider an arbitrary seed $Q=\#^{p}-\#^{q}, p+q=d$, and assume by symmetry that $p \geq q$. Observe that the longest $(m, 1)$-similarity that is not detected by $Q$ is $1^{p-1} 01^{p+q}$ of length $(2 p+q)$. Therefore, we have to minimize $2 p+q=d+p$, and since $p \geq\lceil d / 2\rceil$, the minimum is reached for $p=\lceil d / 2\rceil, q=\lfloor d / 2\rfloor$.

However, for $k \geq 2$, an optimal seed has an asymmetric structure described by the following theorem.

Theorem 1: Let $n$ be an integer and $r=[d / 3]$ ( $[x]$ is the closest integer to $x$ ). For every $k \geq 2$, seed $Q(d)=\#^{d-r}-\#^{r}$ is $k$-optimal among the seeds of $\mathcal{C}_{1}(d)$.

Proof: Again, consider a seed $Q=\#^{p}-\#^{q}, p+q=d$, and assume that $p \geq q$. Consider the longest word $S(k)$ from $\left(1^{*} 0\right)^{k} 1^{*}, k \geq 1$, which is not detected by $Q$ and let $L(k)$ is the 
length of $S(k)$. By the above remark, $S(1)=1^{p-1} 01^{p+q}$ and $L(1)=2 p+q$.

It is easily seen that for every $k, S(k)$ starts either with $1^{p-1} 0$, or with $1^{p+q} 01^{q-1} 0$. Define $L^{\prime}(k)$ to be the maximal length of a word from $\left(1^{*} 0\right)^{k} 1^{*}$ that is not detected by $Q$ and starts with $1^{q-1} 0$. Since prefix $1^{q-1} 0$ implies no additional constraint on the rest of the word, we have $L^{\prime}(k)=q+L(k-1)$. Observe that $L^{\prime}(1)=p+2 q$ (word $1^{q-1} 01^{p+q}$ ). To summarize, we have the following recurrences for $k \geq 2$ :

$$
\begin{aligned}
L^{\prime}(k) & =q+L(k-1), \\
L(k) & =\max \left\{p+L(k-1), p+q+1+L^{\prime}(k-1)\right\},
\end{aligned}
$$

with initial conditions $L^{\prime}(1)=p+2 q, L(1)=2 p+q$.

Two cases should be distinguished. If $p \geq 2 q+1$, then the straightforward induction shows that the first term in (2) is always greater, and we have

$$
L(k)=(k+1) p+q,
$$

and the corresponding longest word is

$$
S(k)=\left(1^{p-1} 0\right)^{k} 1^{p+q} .
$$

If $q \leq p \leq 2 q+1$, then by induction, we obtain

$$
L(k)= \begin{cases}(\ell+1) p+(k+1) q+\ell & \text { if } k=2 \ell, \\ (\ell+2) p+k q+\ell & \text { if } k=2 \ell+1,\end{cases}
$$

and

$$
S(k)= \begin{cases}\left(1^{p+q} 01^{q-1} 0\right)^{\ell} 1^{p+q} & \text { if } k=2 \ell, \\ 1^{p-1} 0\left(1^{p+q} 01^{q-1} 0\right)^{\ell} 1^{p+q} & \text { if } k=2 \ell+1 .\end{cases}
$$

By definition of $L(k)$, seed $\#^{p}-\#^{q}$ detects any word from $\left(1^{*} 0\right)^{k} 1^{*}$ of length $(L(k)+1)$ or more, and this is the tight bound. Therefore, we have to find $p, q$ which minimize $L(k)$. Recall that $p+q=d$, and observe that for $p \geq 2 q+1, L(k)$ (defined by (3)) is increasing on $p$, while for $p \leq 2 q+1, L(k)$ (defined by (5)) is decreasing on $p$. Therefore, both functions reach its minimum when $p=2 q+1$. Therefore, if $d \equiv 1(\bmod 3)$, we obtain $q=\lfloor d / 3\rfloor$ and $p=d-q$. If $d \equiv 0(\bmod 3)$, a routine computation shows that the minimum is reached at 
$q=d / 3, p=2 d / 3$, and if $d \equiv 2(\bmod 3)$, the minimum is reached at $q=\lceil d / 3\rceil, p=d-q$. Putting the three cases together results in $q=[d / 3], p=d-q$.

To illustrate Theorem 1, seed \#\#\#\#-\#\# is optimal among all seeds of weight 6 with one joker. This means that this seed solves the $(m, 2)$-problem for all $m \geq 16$ and this is the smallest possible bound over all seeds of this class. Similarly, this seed solves the ( $m, 3)$-problem for all $m \geq 20$, which is the best possible bound, etc.

\section{B. Regular expansion and contraction of seeds}

We now show that seeds solving larger problems can be obtained from seeds solving smaller problems, and vice versa, using regular expansion and regular contraction operations.

Given a seed $Q$, its $i$-regular expansion $i \otimes Q$ is obtained by multiplying each matching position by $i$. This is equivalent to inserting $i-1$ jokers between every two successive positions along the seed. For example, if $Q=\{0,2,3,5\}$ (or \#-\#\#-\#), then the 2-regular expansion of $Q$ is $2 \otimes Q=\{0,4,6,10\}$ (or \#---\#-\#---\#). Given a family $F$, its $i$-regular expansion $i \otimes F$ is the family obtained by applying the $i$-regular expansion on each seed of $F$.

Lemma 1: If a family $F$ solves an $(m, k)$-problem, then the $(i m,(i+1) k-1)$-problem is solved both by family $F$ and by its $i$-regular expansion $F_{i}=i \otimes F$.

Proof: Consider an $(i m,(i+1) k-1)$-similarity $w$. By the pigeon hole principle, it contains at least one substring of length $m$ with $k$ mismatches or less, and therefore $F$ solves the $(i m,(i+1) k-1)$-problem. On the other hand, consider $i$ disjoint subsequences of $w$ each one consisting of $m$ positions equal modulo $i$. Again, by the pigeon hole principle, at least one of them contains $k$ mismatches or less, and therefore the $(i m,(i+1) k-1)$-problem is solved by $i \otimes F$.

The following lemma is the inverse of Lemma 1, it states that if seeds solving a bigger problem have a regular structure, then a solution for a smaller problem can be obtained by the regular contraction operation, inverse to the regular expansion.

Lemma 2: If a family $F_{i}=i \otimes F$ solves an $(i m, k)$-problem, then $F$ solves both the $(i m, k)$-problem and the $(m,\lfloor k / i\rfloor)$-problem.

Proof: One can even show that $F$ solves the $(i m, k)$-problem with the additional restriction for $F$ to match inside one of the position intervals $[1 . . m],[m+1 . .2 m], \ldots,[(i-$ 
1) $m+1 . . \mathrm{im}]$. This is done by using the bijective mapping from Lemma 11: given an $(i m, k)$ similarity $w$, consider $i$ disjoint subsequences $w_{j}(0 \leq j \leq i-1)$ of $w$ obtained by picking $m$ positions equal to $j$ modulo $i$, and then consider the concatenation $w^{\prime}=w_{1} w_{2} \ldots w_{i-1} w_{0}$.

For every $(i m, k)$-similarity $w^{\prime}$, its inverse image $w$ is detected by $F_{i}$, and therefore $F$ detects $w^{\prime}$ at one of the intervals $[1 . . m],[m+1 . .2 m], \ldots,[(i-1) m+1 . . i m]$. Futhermore, for any $(m,\lfloor k / i\rfloor)$-similarity $v$, consider $w^{\prime}=v^{i}$ and its inverse image $w$. As $w^{\prime}$ is detected by $F_{i}, v$ is detected by $F$.

Example 1: To illustrate the two lemmas above, we give the following example pointed out in [1]. The following two seeds are the only seeds of weight 12 that solve the $(50,5)$-problem:

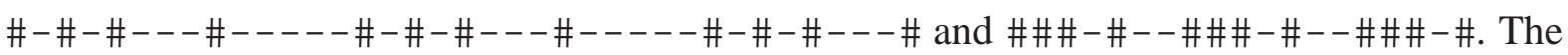
first one is the 2-regular expansion of the second. The second one is the only seed of weight 12 that solves the $(25,2)$-problem.

The regular expansion allows, in some cases, to obtain an efficient solution for a larger problem by reducing it to a smaller problem for which an optimal or a near-optimal solution is known.

\section{Periodic seeds}

In this section, we study seeds with a periodic structure that can be obtained by iterating a smaller seed. Such seeds often turn out to be among maximally weighted seeds solving a given $(m, k)$-problem. Interestingly, this contrasts with the lossy framework where optimal seeds usually have a "random" irregular structure.

Consider two seeds $Q_{1}, Q_{2}$ represented as words over $\{\#,-\}$. In this section, we lift the assumption that a seed must start and end with a matching position. We denote $\left[Q_{1}, Q_{2}\right]^{i}$ the seed defined as $\left(Q_{1} Q_{2}\right)^{i} Q_{1}$. For example, $[\# \# \#-\#,--]^{2}=\# \# \#-\#--\# \# \#-\#--\# \# \#-\#$.

We also need a modification of the $(m, k)$-problem, where $(m, k)$-similarities are considered modulo a cyclic permutation. We say that a seed family $F$ solves a cyclic $(m, k)$-problem, if for every $(m, k)$-similarity $w, F$ detects one of cyclic permutations of $w$. Trivially, if $F$ solves an $(m, k)$-problem, it also solves the cyclic $(m, k)$-problem. To distinguish from a cyclic problem, we call sometimes an $(m, k)$-problem a linear problem.

We first restrict ourselves to the single-seed case. The following lemma demonstrates that iterating smaller seeds solving a cyclic problem allows to obtain a solution for bigger 
problems, for the same number of mismatches.

Lemma 3: If a seed $Q$ solves a cyclic $(m, k)$-problem, then for every $i \geq 0$, the seed $Q_{i}=\left[Q,-^{(m-s(Q))}\right]^{i}$ solves the linear $(m \cdot(i+1)+s(Q)-1, k)$-problem. If $i \neq 0$, the inverse holds too.

Proof: $\Rightarrow$ Consider an $(m \cdot(i+1)+s(Q)-1, k)$-similarity $u$. Transform $u$ into a similarity $u^{\prime}$ for the cyclic $(m, k)$-problem as follows. For each mismatch position $\ell$ of $u$, set 0 at position $(\ell \bmod m)$ in $u^{\prime}$. The other positions of $u^{\prime}$ are set to 1 . Clearly, there are at most $k 0$ 's in $u$. As $Q$ solves the $(m, k)$-cyclic problem, we can find at least one position $j, 1 \leq j \leq m$, such that $Q$ detects $u^{\prime}$ cyclicly.

We show now that $Q_{i}$ matches at position $j$ of $u$ (which is a valid position as $1 \leq j \leq m$ and $\left.s\left(Q_{i}\right)=i m+s(Q)\right)$. As the positions of 1 in $u$ are projected modulo $m$ to matching positions of $Q$, then there is no 0 under any matching element of $Q_{i}$, and thus $Q_{i}$ detects $u$.

$\Leftarrow$ Consider a seed $Q_{i}=\left[Q,-^{(m-s(Q))}\right]^{i}$ solving the $(m \cdot(i+1)+s(Q)-1, k)$-problem. As $i>0$, consider $(m \cdot(i+1)+s(Q)-1, k)$-similarities having all their mismatches located inside the interval $[m, 2 m-1]$. For each such similarity, there exists a position $j, 1 \leq j \leq m$, such that $Q_{i}$ detects it. Note that the span of $Q_{i}$ is at least $m+s(Q)$, which implies that there is either an entire occurrence of $Q$ inside the window $[m, 2 m-1]$, or a prefix of $Q$ matching a suffix of the window and the complementary suffix of $Q$ matching a prefix of the window. This implies that $Q$ solves the cyclic $(m, k)$-problem.

Example 2: Observe that the seed \#\#\#-\# solves the cyclic $(7,2)$-problem. From Lemma 3, this implies that for every $i \geq 0$, the $(11+7 i, 2)$-problem is solved by the seed [\#\#\#-\#,--] ${ }^{i}$ of span $5+7 i$. Moreover, for $i=1,2,3$, this seed is optimal (maximally weighted) over all seeds solving the problem.

By a similar argument based on Lemma 3, the periodic seed [\#\#\#\#\#-\#\#,---] ${ }^{i}$ solves the $(18+11 i, 2)$-problem. Note that its weight grows as $\frac{7}{11} m$ compared to $\frac{4}{7} m$ for the seed from the previous paragraph. However, when $m \rightarrow \infty$, this is not an asymptotically optimal bound, as we will see later.

The $(18+11 i, 3)$-problem is solved by the seed (\#\#\#-\#--\#,---)i , as seed \#\#\#-\#--\# solves the cyclic $(11,3)$-problem. For $i=1,2$, the former is a maximally weighted seed among all solving the $(18+11 i, 3)$-problem. 
One question raised by these examples is whether iterating some seed could provide an asymptotically optimal solution, i.e. a seed of maximal asymptotic weight. The following theorem establishes a tight asymptotic bound on the weight of an optimal seed, for a fixed number of mismatches. It gives a negative answer to this question, as it shows that the maximal weight grows faster than any linear fraction of the similarity size.

Theorem 2: Consider a constant $k$. Let $w(m)$ be the maximal weight of a seed solving the cyclic $(m, k)$-problem. Then $(m-w(m))=\Theta\left(m^{\frac{k-1}{k}}\right)$.

Proof: Note first that all seeds solving cyclic a $(m, k)$-problem can be considered as seeds of span $m$. The number of jokers in any seed $Q$ is then $n=m-w(Q)$. The theorem states that the minimal number of jokers of a seed solving the $(m, k)$-problem is $\Theta\left(m^{\frac{k-1}{k}}\right)$ for every fixed $k$.

Lower bound Consider a cyclic $(m, k)$-problem. The number $D(m, k)$ of distinct cyclic $(m, k)$-similarities satisfies

$$
\frac{\left(\begin{array}{l}
m \\
k
\end{array}\right)}{m} \leq D(m, k)
$$

as every linear $(m, k)$-similarity has at most $m$ cyclicly equivalent ones. Consider a seed $Q$. Let $n$ be the number of jokers in $Q$ and $J_{Q}(m, k)$ the number of distinct cyclic $(m, k)$ similarities detected by $Q$. Observe that $J_{Q}(m, k) \leq\left(\begin{array}{l}n \\ k\end{array}\right)$ and if $Q$ solves the cyclic $(m, k)$ problem, then

$$
D(m, k)=J_{Q}(m, k) \leq\left(\begin{array}{l}
n \\
k
\end{array}\right)
$$

From (7) and (8), we have

$$
\frac{\left(\begin{array}{c}
m \\
k
\end{array}\right)}{m} \leq\left(\begin{array}{l}
n \\
k
\end{array}\right) .
$$

Using the Stirling formula, this gives $n(k)=\Omega\left(m^{\frac{k-1}{k}}\right)$.

Upper bound To prove the upper bound, we construct a seed $Q$ that has no more then $k \cdot m^{\frac{k-1}{k}}$ joker positions and solves the cyclic $(m, k)$-problem.

We start with the seed $Q_{0}$ of span $m$ with all matching positions, and introduce jokers into it in $k$ steps. After step $i$, the obtained seed is denoted $Q_{i}$, and $Q=Q_{k}$.

Let $B=\left\lceil m^{\frac{1}{k}}\right\rceil . Q_{1}$ is obtained by introducing into $Q_{0}$ individual jokers with periodicity $B$ by placing jokers at positions $1, B+1,2 B+1, \ldots$.. At step 2 , we introduce into $Q_{1}$ contiguous intervals of jokers of length $B$ with periodicity $B^{2}$, such that jokers are placed at positions $[1 \ldots B],\left[B^{2}+1 \ldots B^{2}+B\right],\left[2 B^{2}+1 \ldots 2 B^{2}+B\right], \ldots$ 


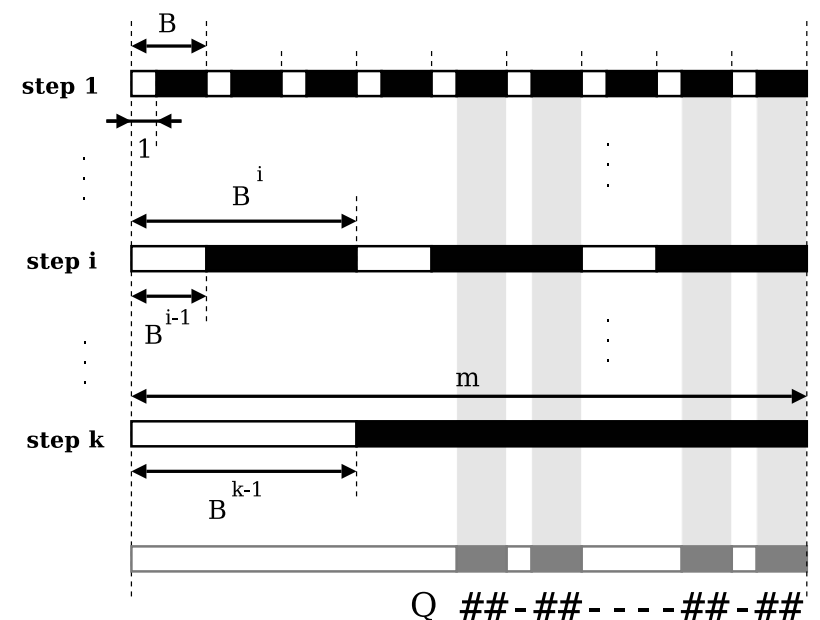

Fig. 1

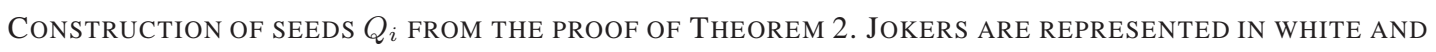
MATCHING POSITIONS IN BLACK.

In general, at step $i(i \leq k)$, we introduce into $Q_{i}$ intervals of $B^{i-1}$ jokers with periodicity $B^{i}$ at positions $\left[1 \ldots B^{i-1}\right],\left[B^{i}+1 \ldots B^{i}+B^{i-1}\right], \ldots$ (see Figure [1).

Note that $Q_{i}$ is periodic with periodicity $B^{i}$. Note also that at each step $i$, we introduce at most $\left\lfloor m^{1-\frac{i}{k}}\right\rfloor$ intervals of $B^{i-1}$ jokers. Moreover, due to overlaps with already added jokers, each interval adds $(B-1)^{i-1}$ new jokers.

This implies that the total number of jokers added at step $i$ is at most $m^{1-\frac{i}{k}} \cdot(B-1)^{i-1} \leq$ $m^{1-\frac{i}{k}} \cdot m^{\frac{1}{k} \cdot(i-1)}=m^{\frac{k-1}{k}}$. Thus, the total number of jokers in $Q$ is less than $k \cdot m^{\frac{k-1}{k}}$.

By induction on $i$, we prove that for any $(m, i)$-similarity $u(i \leq k), Q_{i}$ detects $u$ cyclicly, that is there is a cyclic shift of $Q_{i}$ such that all $i$ mismatches of $u$ are covered with jokers introduced at steps $1, \ldots, i$.

For $i=1$, the statement is obvious, as we can always cover the single mismatch by shifting $Q_{1}$ by at most $(B-1)$ positions. Assuming that the statement holds for $(i-1)$, we show now that it holds for $i$ too. Consider an $(m, i)$-similarity $u$. Select one mismatch of $u$. By induction hypothesis, the other $(i-1)$ mismatches can be covered by $Q_{i-1}$. Since $Q_{i-1}$ has period $B^{i-1}$ and $Q_{i}$ differs from $Q_{i-1}$ by having at least one contiguous interval of $B^{i-1}$ jokers, we can always shift $Q_{i}$ by $j \cdot B^{i-1}$ positions such that the selected mismatch falls into this interval. This shows that $Q_{i}$ detects $u$. We conclude that $Q$ solves the cyclic 
$(m, i)$-problem.

Using Theorem 2, we obtain the following bound on the number of jokers for the linear $(m, k)$-problem.

Lemma 4: Consider a constant $k$. Let $w(m)$ be the maximal weight of a seed solving the linear $(m, k)$-problem. Then $(m-w(m))=\Theta\left(m^{\frac{k}{k+1}}\right)$.

Proof: To prove the upper bound, we construct a seed $Q$ that solves the linear $(m, k)$ problem and satisfies the asymptotic bound. Consider some $l<m$ that will be defined later, and let $P$ be a seed that solves the cyclic $(l, k)$-problem. Without loss of generality, we assume $s(P)=l$.

For a real number $e \geq 1$, define $P^{e}$ to be the maximally weighted seed of span at most $l^{e}$ of the form $P^{\prime} \cdot P \cdots P \cdot P^{\prime \prime}$, where $P^{\prime}$ and $P^{\prime \prime}$ are respectively a suffix and a prefix of $P$. Due to the condition of maximal weight, $w\left(P^{e}\right) \geq e \cdot w(P)$.

We now set $Q=P^{e}$ for some real $e$ to be defined. Observe that if $e \cdot l \leq m-l$, then $Q$ solves the linear $(m, k)$-problem. Therefore, we set $e=\frac{m-l}{l}$.

From the proof of Theorem 2 , we have $l-w(P) \leq k \cdot l^{\frac{k-1}{k}}$. We then have

$$
w(Q)=e \cdot w(P) \geq \frac{m-l}{l} \cdot\left(l-k \cdot l^{\frac{k-1}{k}}\right) .
$$

If we set

$$
l=m^{\frac{k}{k+1}},
$$

we obtain

$$
m-w(Q) \leq(k+1) m^{\frac{k}{k+1}}-k m^{\frac{k-1}{k+1}}
$$

and as $k$ is constant,

$$
m-w(Q)=\mathcal{O}\left(m^{\frac{k}{k+1}}\right) .
$$

The lower bound is obtained similarly to Theorem 2. Let $Q$ be a seed solving a linear $(m, k)$-problem, and let $n=m-w(Q)$. From simple combinatorial considerations, we have

$$
\left(\begin{array}{c}
m \\
k
\end{array}\right) \leq\left(\begin{array}{l}
n \\
k
\end{array}\right) \cdot(m-s(Q)) \leq\left(\begin{array}{l}
n \\
k
\end{array}\right) \cdot n,
$$

which implies $n=\Omega\left(m^{\frac{k}{k+1}}\right)$ for constant $k$.

The following simple lemma is also useful for constructing efficient seeds. 
Lemma 5: Assume that a family $F$ solves an $(m, k)$-problem. Let $F^{\prime}$ be the family obtained from $F$ by cutting out $l$ characters from the left and $r$ characters from the right of each seed of $F$. Then $F^{\prime}$ solves the $(m-r-l, k)$-problem.

Example 3: The $(9+7 i, 2)$-problem is solved by the seed [\#\#\#,-\#--] $]^{i}$ which is optimal for $i=1,2,3$. Using Lemma 5, this seed can be immediately obtained from the seed [\#\#\#-\#,-- $]^{i}$ from Example \&, solving the $(11+7 i, 2)$-problem.

We now apply the above results for the single seed case to the case of multiple seeds.

For a seed $Q$ considered as a word over $\{\#,-\}$, we denote by $Q_{[i]}$ its cyclic shift to the left by $i$ characters. For example, if $Q=\# \# \# \#-\#-\# \#--$, then $Q_{[5]}=\#-\# \#--\# \# \# \#-$. The following lemma gives a way to construct seed families solving bigger problems from an individual seed solving a smaller cyclic problem.

Lemma 6: Assume that a seed $Q$ solves a cyclic $(m, k)$-problem and assume that $s(Q)=m$ (otherwise we pad $Q$ on the right with $(m-s(Q))$ jokers). Fix some $i>1$. For some $L>0$, consider a list of $L$ integers $0 \leq j_{1}<\cdots<j_{L}<m$, and define a family of seeds $F=<\left\|\left(Q_{\left[j_{l}\right]}\right)^{i}\right\|>_{l=1}^{L}$, where $\left\|\left(Q_{\left[j_{l}\right]}\right)^{i}\right\|$ stands for the seed obtained from $\left(Q_{\left[j_{l}\right]}\right)^{i}$ by deleting the joker characters at the left and right edges. Define $\delta(l)=\left(\left(j_{l-1}-j_{l}\right) \bmod m\right)$ (or, alternatively, $\left.\delta(l)=\left(\left(j_{l}-j_{l-1}\right) \bmod m\right)\right)$ for all $l, 1 \leq l \leq L$. Let $m^{\prime}=\max \left\{s\left(\left\|\left(Q_{\left[j_{l}\right]}\right)^{i}\right\|\right)+\right.$ $\delta(l)\}_{l=1}^{L}-1$. Then $F$ solves the $\left(m^{\prime}, k\right)$-problem.

Proof: The proof is an extension of the proof of Lemma 3. Here, the seeds of the family are constructed in such a way that for any instance of the linear $\left(m^{\prime}, k\right)$-problem, there exists at least one seed that satisfies the property required in the proof of Lemma 3 and therefore matches this instance.

In applying Lemma 6, integers $j_{l}$ are chosen from the interval $[0, m]$ in such a way that values $s\left(\left\|\left(Q\left[j_{l}\right]\right)^{i}\right\|\right)+\delta(l)$ are closed to each other. We illustrate Lemma 6 with two examples that follow.

Example 4: Let $m=11, k=2$. Consider the seed $Q=\# \# \# \#-\#-\# \#--$ solving the cyclic $(11,2)$-problem. Choose $i=2, L=2, j_{1}=0, j_{2}=5$. This gives two seeds $Q_{1}=\left\|\left(Q_{[0]}\right)^{2}\right\|=$ \#\#\#\#-\#-\#\#--\#\#\#\#-\#-\#\# and $Q_{2}=\left\|\left(Q_{[5]}\right)^{2}\right\|=\#-\# \#--\# \# \# \#-\#-\# \#--\# \# \#$ of span 20 and 21 respectively, $\delta(1)=6$ and $\delta(2)=5 \cdot \max \{20+6,21+5\}-1=25$. Therefore, family $F=\left\{Q_{1}, Q_{2}\right\}$ solves the (25,2)-problem. 
Example 5: Let $m=11, k=3$. The seed $Q=\# \# \#-\#--\#---$ solving the cyclic $(11,3)$ problem. Choose $i=2, L=2, j_{1}=0, j_{2}=4$. The two seeds are $Q_{1}=\left\|\left(Q_{[0]}\right)^{2}\right\|=$ \#\#\#-\#--\#---\#\#\#-\#--\# (span 19) and $Q_{2}=\left\|\left(Q_{[4]}\right)^{2}\right\|=\#--\#---\# \# \#-\#--\#---\# \# \#$ $(\operatorname{span} 21)$, with $\delta(1)=7$ and $\delta(2)=4 \cdot \max \{19+7,21+4\}-1=25$. Therefore, family $F=\left\{Q_{1}, Q_{2}\right\}$ solves the $(25,3)$-problem.

\section{Heuristic seed design}

Results of Sections $\mathbb{D - A}[\mathrm{IV}-\mathrm{Q}$ allow one to construct efficient seed families in certain cases, but still do not allow a systematic seed design. Recently, linear programming approaches to designing efficient seed families were proposed in [19] and in [18], respectively for DNA and protein similarity search. However, neither of these methods aims at constructing lossless families.

In this section, we outline a heuristic genetic programming algorithm for designing lossless seed families. The algorithm will be used in the experimental part of this work, that we present in the next section. Note that this algorithm uses dynamic programming algorithms of Section III. Since the algorithm uses standard genetic programming techniques, we give only a high-level description here without going into all details.

The algorithm tries to iteratively improve characteristics of a population of seed families until it finds a small family that detects all $(m, k)$-similarities (i.e. is lossless). The first step of each iteration is based on screening current families against a set of difficult similarities that are similarities that have been detected by fewer families. This set is continually reordered and updated according to the number of families that don't detect those similarities. For this, each set is stored in a tree and the reordering is done using the list-as-a-tree principle [20]: each time a similarity is not detected by a family, it is moved towards the root of the tree such that its height is divided by two.

For those families that pass through the screening, the number of undetected similarities is computed by the dynamic programming algorithm of Section III-B. The family is kept if it produces a smaller number than the families currently known. An undetected similarity obtained during this computation is added as a leaf to the tree of difficult similarities.

To detect seeds to be improved inside a family, we compute the contribution of each seed by the dynamic programming algorithm of Section III-C. The seeds with the least contribution 
are then modified with a higher probability. In general, the population of seed families is evolving by mutating and crossing over according to the set of similarities they do not detect. Moreover, random seed families are regularly injected into the population in order to avoid local optima.

The described heuristic procedure often allows efficient or even optimal solutions to be computed in a reasonable time. For example, in ten runs of the algorithm, we found 3 of the 6 existing families of two seeds of weight 14 solving the $(25,2)$-problem. The whole computation took less than 1 hour, compared to a week of computation needed to exhaustively test all seed pairs. Note that the randomized-greedy approach (incremental completion of the seed set by adding the best random seed) applied a dozen of times to the same problem yielded only sets of three and sometimes four, but never two seeds, taking about 1 hour at each run.

\section{EXPERIMENTS}

We describe two groups of experiments that we made. The first one concerns the design of efficient seed families, and the second one applies a multi-seed lossless filtration to the identification of unique oligos in a large set of EST sequences.

\section{Seed design experiments}

We considered several $(m, k)$-problems. For each problem, and for a fixed number of seeds in the family, we computed families solving the problem and realizing the largest possible seed weight (under a natural assumption that all seeds in a family have the same weight). We also kept track of the ways (periodic seeds, genetic programming heuristics, exhaustive search) in which those families can be computed.

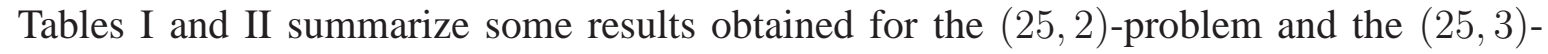
problem respectively. Families of periodic seeds (that can be found using Lemma 6) are marked with ${ }^{p}$, those that are found using a genetic algorithm are marked with ${ }^{g}$, and those which are obtained by an exhaustive search are marked with ${ }^{e}$. Only in this latter case, the families are guaranteed to be optimal. Families of periodic seeds are shifted according to their construction (see Lemma 6). 
TABLE I

SEED FAMILIES FOR $(25,2)$-PROBLEM

\begin{tabular}{|c|c|c|c|}
\hline size & weight & family seeds & $\delta$ \\
\hline 1 & $12^{e, p, g}$ & \#\#\#-\#--\#\#\#-\#--\#\#\#-\# & $5.96 \cdot 10^{-8}$ \\
\hline \multirow[t]{2}{*}{2} & $14^{e, p, g}$ & \#\#\#\#-\#-\#\#--\#\#\#\#-\#-\#\# & $7.47 \cdot 10^{-9}$ \\
\hline & & \#-\#\#--\#\#\#\#-\#-\#\#--\#\#\#\# & \\
\hline \multirow[t]{3}{*}{3} & $15^{p}$ & \#--\#\#-\#-\#\#\#\#\#\#--\#\#-\#-\#\# & $2.80 \cdot 10^{-9}$ \\
\hline & & \#-\#\#\#\#\#\#--\#\#-\#-\#\#\#\#\# & \\
\hline & & \#\#\#\#--\#\#-\#-\#\#\#\#\#\#--\#\# & \\
\hline \multirow[t]{4}{*}{4} & $16^{p}$ & 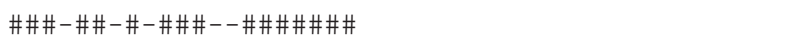 & $9.42 \cdot 10^{-10}$ \\
\hline & & \#\#-\#-\#\#\#--\#\#\#\#\#\#\#-\#\#-\# & \\
\hline & & \#\#\#--\#\#\#\#\#\#\#-\#\#-\#-\#\#\# & \\
\hline & & \#\#\#\#\#\#\#-\#\#-\#-\#\#\#--\#\#\# & \\
\hline \multirow[t]{6}{*}{6} & $17^{p}$ & \#\#-\#-\#\#--\#\#\#\#\#\#\#-\#\#\#\#-\# & $3.51 \cdot 10^{-10}$ \\
\hline & & 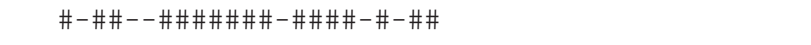 & \\
\hline & & 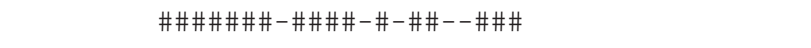 & \\
\hline & & 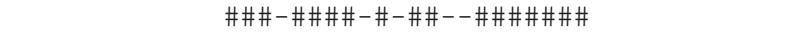 & \\
\hline & & 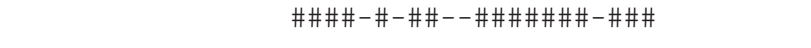 & \\
\hline & & 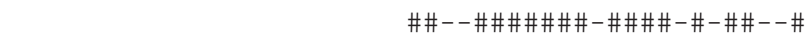 & \\
\hline
\end{tabular}

TABLE II

SEED FAMILIES FOR $(25,3)$-PROBLEM

\begin{tabular}{|c|c|c|c|}
\hline size & weight & family seeds & $\delta$ \\
\hline 1 & $8^{e, p, g}$ & \#\#\#-\#------\#\#\#-\# & $1.53 \cdot 10^{-5}$ \\
\hline \multirow[t]{2}{*}{2} & $10^{p}$ & \#\#\#\#-\#-\#\#--\#--- \#\# & $1.91 \cdot 10^{-6}$ \\
\hline & & \#\#--\#--- \#\#\#\#-\#-\#\# & \\
\hline \multirow[t]{3}{*}{3} & $11^{p}$ & \#--- \#\#\#\#-\#-\#\#--\#---\#\# & $7.16 \cdot 10^{-7}$ \\
\hline & & \#\#\#-\#-\#\#--\#----\#\#\#\# & \\
\hline & & \#\#--\#----\#\#\#\#-\#-\#\#--\# & \\
\hline \multirow[t]{4}{*}{4} & $12^{p}$ & \#---\#\#\#\#-\#-\#\#---\#----\#\#\# & $2.39 \cdot 10^{-7}$ \\
\hline & & \#\#\#-\#-\#\#--\#---\#\#\#\#-\# & \\
\hline & & \#-\#\#--\#----\#\#\#\#-\#-\#\#--\# & \\
\hline & & 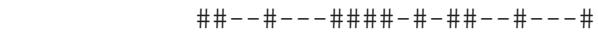 & \\
\hline
\end{tabular}

Moreover, to compare the selectivity of different families solving a given $(m, k)$-problem, we estimated the probability $\delta$ for at least one of the seeds of the family to match at a given position of a uniform Bernoulli four-letter sequence. This has been done using the inclusion-exclusion formula.

Note that the simple fact of passing from a single seed to a two-seed family results in a considerable gain in efficiency: in both examples shown in the tables there a change of about 
one order magnitude in the selectivity estimator $\delta$.

\section{Oligo selection using multi-seed filtering}

An important practical application of lossless filtration is the selection of reliable oligonucleotides for DNA micro-array experiments. Oligonucleotides (oligos) are small DNA sequences of fixed size (usually ranging from 10 to 50) designed to hybridize only with a specific region of the genome sequence. In micro-array experiments, oligos are expected to match ESTs that stem from a given gene and not to match those of other genes. As the first approximation, the problem of oligo selection can then be formulated as the search for strings of a fixed length that occur in a given sequence but do not occur, within a specified distance, in other sequences of a given (possibly very large) sample. Different approaches to this problem apply different distance measures and different algorithmic techniques [21], [22], [23], [24]. The experiments we briefly present here demonstrate that the multi-seed filtering provides an efficient computation of candidate oligonucleotides. These should then be further processed by complementary methods in order to take into account other physicochemical factors occurring in hybridisation, such as the melting temperature or the possible hairpin structure of palindromic oligos.

Here we adopt the formalization of the oligo selection problem as the problem of identifying in a given sequence (or a sequence database) all substrings of length $m$ that have no occurrences elsewhere in the sequence within the Hamming distance $k$. The parameters $m$ and $k$ were set to 32 and 5 respectively. For the $(32,5)$-problem, different seed families were designed and their selectivity was estimated. Those are summarized in the table in Figure 2, using the same conventions as in Tables $\llbracket$ and $\llbracket$ above. The family composed of 6 seeds of weight 11 was selected for the filtration experiment (shown in Figure 2).

The filtering has been applied to a database of rice EST sequences composed of 100015 sequences for a total length of 42,845,242 bp 円. Substrings matching other substrings with 5 substitution errors or less were computed. The computation took slightly more than one hour on a Pentium ${ }^{\mathrm{TM}} 43 \mathrm{GHz}$ computer. Before applying the filtering using the family for the $(32,5)$-problem, we made a rough pre-filtering using one spaced seed of weight 16 to detect,

\footnotetext{
${ }^{1}$ source : http://bioserver.myongji.ac.kr/ricemac.html, The Korea Rice Genome Database
} 


\begin{tabular}{|c|c|c|}
\hline family size & weight & $\delta$ \\
\hline 1 & $7^{e}$ & $6.10 \cdot 10^{-5}$ \\
2 & $8^{e}$ & $3.05 \cdot 10^{-5}$ \\
3 & $9^{e}$ & $1.14 \cdot 10^{-5}$ \\
4 & $10^{g}$ & $3.81 \cdot 10^{-6}$ \\
6 & $11^{g}$ & $1.43 \cdot 10^{-6}$ \\
10 & $12^{g}$ & $5.97 \cdot 10^{-7}$ \\
\hline
\end{tabular}

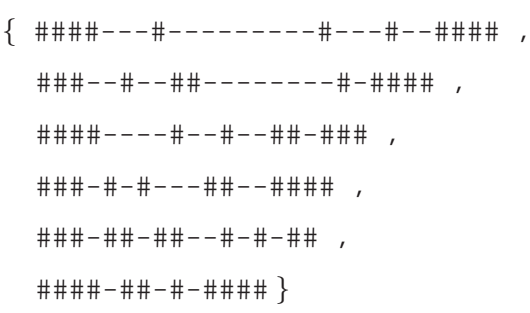

Fig. 2

COMPUTED SEED FAMILIES FOR THE $(32,5)$-PROBLEM AND THE CHOSEN FAMILY (6 SEEDS OF WEIGHT 11$)$

with a high selectivity, almost identical regions. $65 \%$ of the database has been discarded by this pre-filtering. Another $22 \%$ of the database has been filtered out using the chosen seed family, leaving the remaining $13 \%$ as oligo candidates.

\section{CONCLUSiON}

In this paper, we studied a lossless filtration method based on multi-seed families and demonstrated that it represents an improvement compared to the single-seed approach considered in [1]. We showed how some important characteristics of seed families can be computed using the dynamic programming. We presented several combinatorial results that allow one to construct efficient families composed of seeds with a periodic structure. Finally, we described a large-scale computational experiment of designing reliable oligonucleotides for DNA microarrays. The obtained experimental results provided evidence of the applicability and efficiency of the whole method.

The results of Sections IV-A-IV-C establish several combinatorial properties of seed families, but many more of them remain to be elucidated. The structure of optimal or nearoptimal seed families can be reduced to number-theoretic questions, but this relation remains to be clearly established. In general, constructing an algorithm to systematically design seed families with quality guarantee remains an open problem. Some complexity issues remain open too: for example, what is the complexity of testing if a single seed is lossless for given $m, k$ ? Section III implies a time bound exponential on the number of jokers. Note that for multiple seeds, computing the number of detected similarities is NP-complete [16, Section 3.1].

Another direction is to consider different distance measures, especially the Levenstein 
distance, or at least to allow some restricted insertion/deletion errors. The method proposed in [25] does not seem to be easily generalized to multi-seed families, and a further work is required to improve lossless filtering in this case.

Acknowledgements: G. Kucherov and L. Noé have been supported by the French Action Spécifique "Algorithmes et Séquences" of CNRS. A part of this work has been done during a stay of M. Roytberg at LORIA, Nancy, supported by INRIA. M. Roytberg has been supported by the Russian Foundation for Basic Research (project nos. 03-04-49469, 02-07-90412) and by grants from the RF Ministry for Industry, Science, and Technology (20/2002, 5/2003) and NWO.

\section{REFERENCES}

[1] S. Burkhardt and J. Kärkkäinen, "Better filtering with gapped q-grams," Fundamenta Informaticae, vol. 56, no. 1-2, pp. 51-70, 2003, preliminary version in Combinatorial Pattern Matching 2001.

[2] G. Navarro and M. Raffinot, Flexible Pattern Matching in Strings - Practical on-line search algorithms for texts and biological sequences. Cambridge University Press, 2002.

[3] S. Altschul, T. Madden, A. Schäffer, J. Zhang, Z. Zhang, W. Miller, and D. Lipman, "Gapped BLAST and PSI-BLAST: a new generation of protein database search programs," Nucleic Acids Research, vol. 25, no. 17, pp. 3389-3402, 1997.

[4] B. Ma, J. Tromp, and M. Li, "PatternHunter: Faster and more sensitive homology search," Bioinformatics, vol. 18, no. 3, pp. 440-445, 2002.

[5] S. Schwartz, J. Kent, A. Smit, Z. Zhang, R. Baertsch, R. Hardison, D. Haussler, and W. Miller, "Human-mouse alignments with BLASTZ," Genome Research, vol. 13, pp. 103-107, 2003.

[6] L. Noé and G. Kucherov, "Improved hit criteria for DNA local alignment," BMC Bioinformatics, vol. 5, no. 149, october 2004 .

[7] P. Pevzner and M. Waterman, "Multiple filtration and approximate pattern matching," Algorithmica, vol. 13, pp. 135-154, 1995.

[8] A. Califano and I. Rigoutsos, "Flash: A fast look-up algorithm for string homology," in Proceedings of the 1st International Conference on Intelligent Systems for Molecular Biology, July 1993, pp. 56-64.

[9] J. Buhler, "Provably sensitive indexing strategies for biosequence similarity search," in Proceedings of the 6th Annual International Conference on Computational Molecular Biology (RECOMB02), Washington, DC (USA). ACM Press, April 2002, pp. 90-99.

[10] U. Keich, M. Li, B. Ma, and J. Tromp, “On spaced seeds for similarity search,” Discrete Applied Mathematics, vol. 138, no. 3, pp. 253-263, 2004.

[11] J. Buhler, U. Keich, and Y. Sun, "Designing seeds for similarity search in genomic DNA," in Proceedings of the 7th Annual International Conference on Computational Molecular Biology (RECOMB03), Berlin (Germany). ACM Press, April 2003, pp. 67-75. 
[12] B. Brejova, D. Brown, and T. Vinar, "Vector seeds: an extension to spaced seeds allows substantial improvements in sensitivity and specificity," in Proceedings of the 3rd International Workshop in Algorithms in Bioinformatics (WABI), Budapest (Hungary), ser. Lecture Notes in Computer Science, G. Benson and R. Page, Eds., vol. 2812. Springer, September 2003, pp. 39-54.

[13] G. Kucherov, L. Noé, and Y. Ponty, "Estimating seed sensitivity on homogeneous alignments," in Proceedings of the IEEE 4th Symposium on Bioinformatics and Bioengineering (BIBE2004), May 19-21, 2004, Taichung (Taiwan), ser. the IEEE 4th Symposium on Bioinformatics and Bioengineering - BIBE'2004. IEEE Computer Society Press, April 2004, pp. 387-394.

[14] K. Choi and L. Zhang, "Sensitivity analysis and efficient method for identifying optimal spaced seeds," Journal of Computer and System Sciences, vol. 68, pp. 22-40, 2004.

[15] M. Csürös, "Performing local similarity searches with variable lenght seeds," in Proceedings of the 15th Annual Combinatorial Pattern Matching Symposium (CPM), Istanbul (Turkey), ser. Lecture Notes in Computer Science,

S. Sahinalp, S. Muthukrishnan, and U. Dogrusoz, Eds., vol. 3109. Springer Verlag, 2004, pp. 373-387.

[16] M. Li, B. Ma, D. Kisman, and J. Tromp, "PatternHunter II: Highly sensitive and fast homology search," Journal of Bioinformatics and Computational Biology, vol. 2, no. 3, pp. 417-440, September 2004.

[17] Y. Sun and J. Buhler, "Designing multiple simultaneous seeds for DNA similarity search," in Proceedings of the 8th Annual International Conference on Research in Computational Molecular Biology (RECOMB 2004). ACM Press, March 2004, pp. 76-84.

[18] D. G. Brown, "Multiple vector seeds for protein alignment," in Proceedings of the 4th International Workshop on Algorithms in Bioinformatics (WABI), September 2004, Bergen (Norway), ser. Lecture Notes in Bioinformatics, I. Jonassen and J. Kim, Eds., vol. 3240. Springer Verlag, 2004, pp. 170-181.

[19] J. Xu, D. Brown, M. Li, and B. Ma, “Optimizing multiple spaced seeds for homology search,” in Proceedings of the 15th Symposium on Combinatorial Pattern Matching, Istambul (Turkey), ser. Lecture Notes in Computer Science, S. Sahinalp, S. Muthukrishnan, and U. Dogrusoz, Eds., vol. 3109, 2004, pp. 47-58.

[20] J. Oommen and J. Dong, "Generalized swap-with-parent schemes for self-organizing sequential linear lists," in Proceedings of the 1997 International Symposium on Algorithms and Computation (ISAAC'97), Singapore, ser. Lecture Notes in Computer Science, vol. 1350. Springer, December 17-19 1997, pp. 414-423.

[21] F. Li and G. Stormo, "Selection of optimal DNA oligos for gene expression arrays," Bioinformatics, vol. 17, pp. 1067-1076, 2001.

[22] L. Kaderali and A. Schliep, "Selecting signature oligonucleotides to identify organisms using DNA arrays," Bioinformatics, vol. 18, no. 10, pp. 1340-1349, 2002.

[23] S. Rahmann, "Fast large scale oligonucleotide selection using the longest common factor approach," Journal of Bioinformatics and Computational Biology, vol. 1, no. 2, pp. 343-361, 2003.

[24] J. Zheng, T. Close, T. Jiang, and S. Lonardi, "Efficient selection of unique and popular oligos for large EST databases," in Proceedings of the 14th Annual Combinatorial Pattern Matching Symposium (CPM), 2003, Morelia (Mexico), ser. Lecture Notes in Computer Science, vol. 2676. Springer Verlag, 2003, pp. 273-283.

[25] S. Burkhardt and J. Karkkainen, "One-gapped q-gram filters for Levenshtein Distance," in Proceedings of the 13th Symposium on Combinatorial Pattern Matching (CPM'02), vol. 2373. Springer, 2002, pp. 225-234. 